\title{
Long term clinical and dermoscopic follow-up of a child with a Spitz nevus"
}

\author{
Massimiliano Scalvenzi ${ }^{\#}$, Maria Grazia Francia, Franco Palmisano, Claudia Costa
}

Department of Dermatology, University of Naples Federico II, Naples, Italy

Email: ${ }^{\text {scalvenz@unina.it }}$

Received 31 May 2012; revised 17 July 2012; accepted 25 July 2012

\begin{abstract}
Background: Spitz nevus is uncommon, benign melanocytic neoplasm that may show some clinical, dermoscopical and histological features of melanoma. It occurs often in childhood, but may appear also in early adulthood. Rare congenital cases have been reported in literature. It is frequently located on the face and the lower extremities, but in some cases may appears on the trunk. Methods: We report a case of a 9-year-old girl presented to our Dermatology Unit because of the presence of a pigmented lesion on her right leg, $4 \mathrm{~mm}$ in diameter, which was clinically and dermoscopically diagnosed as Spitz nevus. We described the clinical and dermoscopic features that we observed every six months, over 11 years follow-up period. Objective: Our observation show that the globular, the starburst, the homogeneous patterns and diffuse brown colour with diffuse hypopigmented areas may be the different expression that correspond to possible evolutionary phases of pigmented Spitz nevus.
\end{abstract}

Keywords: Spitz Nevus; Evolution; Dermoscopy

\section{INTRODUCTION}

Spitz nevus in its classic clinical appearance is described as a rapidly growing, pink or flesh-coloured papule or nodule of the face or the lower extremities in childhood; however brown and even black pigmentation is common as well. Described for the first time by Sophie Spitz in 1948 as melanoma of childhood, is now considered as a benign melanocytic neoplasm.

In the last 20 years dermoscopy has been increasingly used as a non invasive diagnostic technique for the in vivo observation of pigmented skin lesions. Dermoscopy is a very useful method in the study of Spitz nevi in order to understand its evolution, which until now was unknown, and subsequently this will lead to a better man-

\footnotetext{
${ }^{*}$ Conflict of Interest: None.

${ }^{\#}$ Corresponding author.
}

agement [1].

Specific dermoscopy criteria have been described for differentiating Spitz nevi from cutaneous melanoma [2]. Spitz nevi can be easily identified by a prominent symmetrical starburst or globular pattern, with a central, bizarre or reticular depigmentation, and a rim of brown globules at the periphery, which in some instances may mimic pseudopods. In addition, Spitz nevi can often show a pattern characterized by an uneven distribution of colours and structures, and an irregular diffuse pigmentation resembling blue-white veil or irregular extensions (black blotches) [2].

In contrast cutaneous melanoma is asymmetrical and irregularly pigmented with variable combinations of broadened pigmented network, blue-white veil, irregular black dots or brown globules, peripheral depigmentation, irregular extensions and radial streaming at the edge of the lesion.

\section{CASE REPORT}

A 9-year-old girl was examinated at our Dermatology Unit for the presence of a pigmented lesion located on her left leg, $4 \mathrm{~mm}$ in diameter, that at the dermoscopic examination revealed the typical features of a pigmented Spitz nevus consisting of a central prominent grey-blue pigmentation and a rim of peripheral large blue-black globules (Figure 1). Because of the dermoscopic pattern strongly suggestive of Spitz nevus no biopsy were performed.

Dermoscopic images have been made with Nikon Coolpix 4500 digital camera (4.0 mega pixels $4 \times$ zoom) and Dermlite foto (3GEN).

Six months later, the lesion enlarged, measuring 6.3 $\mathrm{mm}$ in its largest diameter; dermoscopy showed a starburst pattern with radial streaks regularly distributed over the entire lesion, that was unchanged during the next two years follow-up (Figure 2).

In March 2004, a homogeneous pattern with blue-brown pigmentation in the center of lesion was identified and has still been observed for the following 2 examinations.

Since March 2005 the pattern has been stable, while 


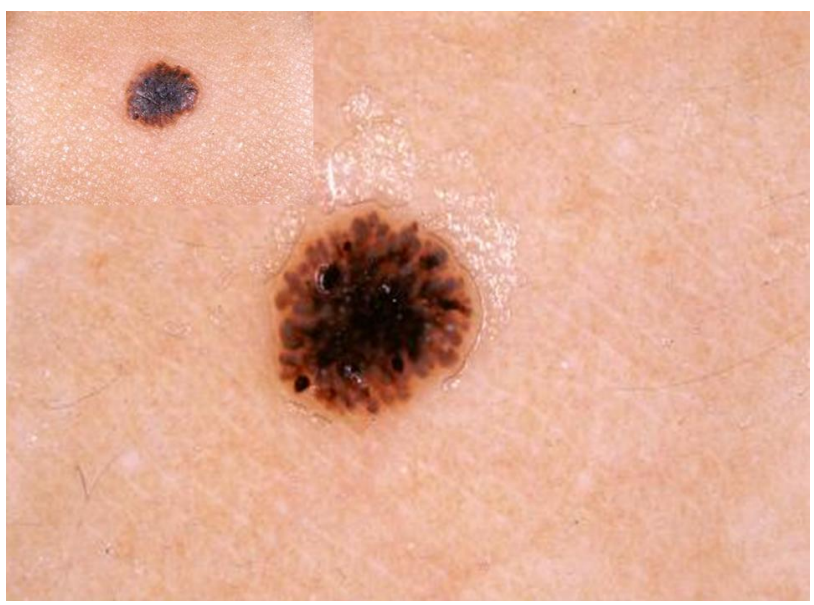

Figure 1. Clinical and dermoscopic typical features of pigmented Spitz nevus consisting of a central prominent grey-blue pigmentation and a rim of peripheral large blue-black globules.



Figure 2. Clinical and dermoscopic features after six month showed a starburst pattern with radial streaks regularly distributed over the entire lesion.

less intensity of the blue-white structures and pigmentation was seen. From June 2006 the lesion showed a homogeneous brown pigmentation with a further reduction of the blue-white structures (Figure 3). In September 2007 we observed at dermoscopic examination the complete regression of the blue-white structures and the presence of multiphocal central hypopigmented areas, and has still been observed for the following follow-up.

In November 2008 the lesion showed only remnants of light-brown pigmentation with large hypopigmented areas (Figure 4).

From November 2008 to May 2012 the lesion showed a further reduction of the light-brown pigmentation (Figure 5).

\section{DISCUSSION}

The natural history of melanocytic nevi is poorly understood

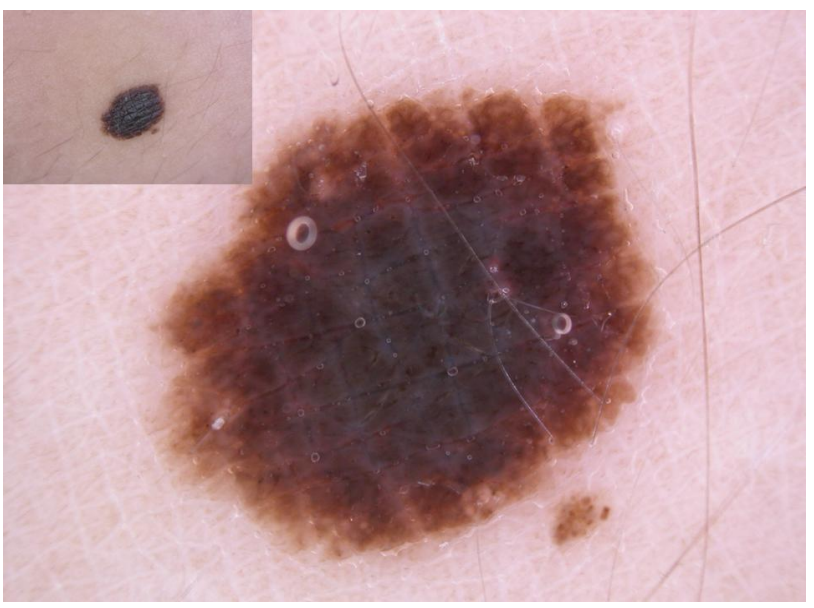

Figure 3. Clinical and dermoscopic features after three years of follow-up showed a homogeneous brown pigmentation with more reduction of blue-white structures.

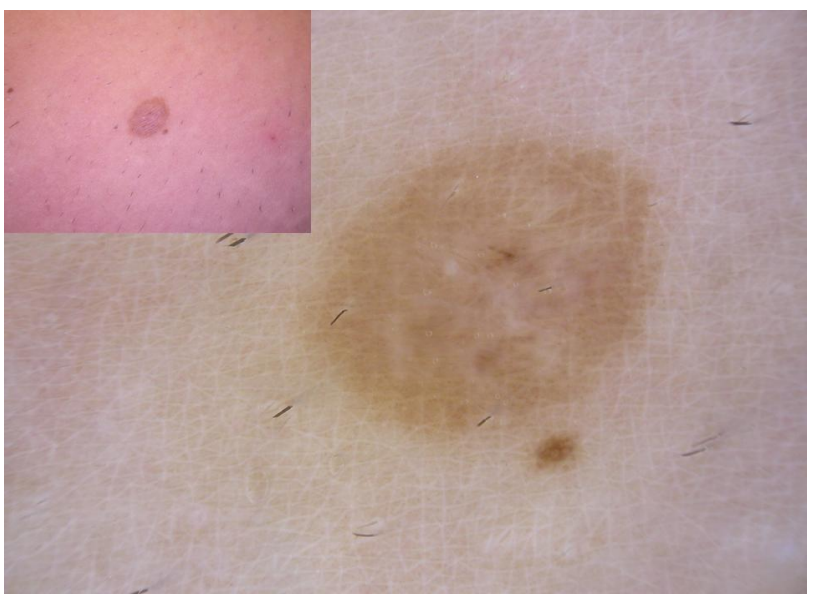

Figure 4. Clinical and dermoscopic features after eight years of follow-up showed only remnants of light-brown pigmentation with large hypopigmented areas.

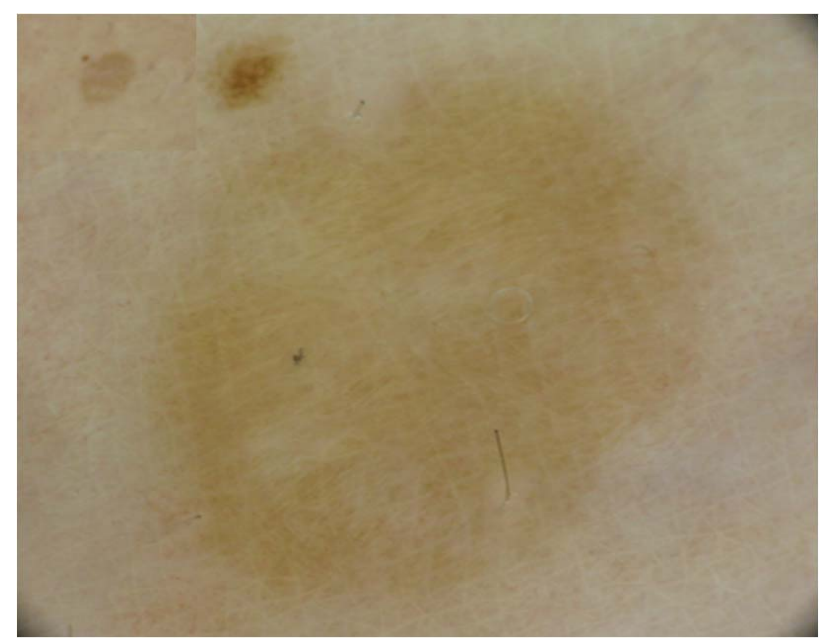

Figure 5. Clinical and dermoscopic features after eleven years of follow-up showed only a further reduction of light-brown pigmentation. 
owing the lack of follow-up studies investigating their long-term evolution.

Spitz nevus is a solitary dome-shaped papule, measuring up to $1 \mathrm{~cm}$ in diameter. Its surface is smooth or keratotic/verrucous and occasionally even papillomatous. Although initially described as a non-pigmented lesion, recent data underline that Spitz nevi are brown to black in $80 \%$ of histological examinated cases. Large $(>1 \mathrm{~cm})$ nodular and/or ulcerated lesions must be always regarded as worrisome even in childhood.

Six main dermoscopic patterns can be described in Spitz nevus: vascular, globular, reticular, starburst, polymorphous and homogeneous [3]. The vascular pattern is characterised by dotted vessels, which are responsible of its pink colour, regularly distributed throughout the lesion. The globular pattern shows the presence of brown to black globules, large and regularly distributed at the periphery. They are most frequent in pigmented Spitz nevus where often they are fused with the central body of the lesion. The reticular pattern shows a heavy pigmentation rise to a regular black network, which rest above the lesion and can be removed by tape stripping. The starburst pattern is characterised by radial projections, also called streaks, that from the centre of lesion came to the periphery. Several of this dermoscopic features can be simultaneously present in the lesion with an irregular distribution in the polymorphous pattern also known as "melanoma-like" pattern. Most of this different dermoscopic pattern simply corresponds to different phases of the natural evolution of Spitz nevus.

Spitz nevus must be differentiated from viral warts, pyogenic granuloma, lymphoid infiltration of the skin, verrucous epidermal nevus, etc. The uniform distribution of vascular pattern in Spitz nevus can also help in the differential diagnosis with amelanotic melanoma. Differential diagnosis of pigmented Spitz nevus may include hyperpigmented Clark nevus and melanoma [4,5]. The occurrence of an atypical dermoscopic pattern in Spitz nevus is well recognized as is the occurrence of melanoma showing very few or no dermoscopic features suggestive of malignancy but exhibiting either the globular or the starburst pattern.

There is no consensus about the benign nature of Spitz nevus, but its natural history is not fully understood and the histopathological differentiation from "spitzoid lesions" as spitzoid melanoma, is often difficult as the management of this lesions.

There are different choices of management [6]: Spitz nevus appearing up to the age of 12 years that can be easily diagnosed and managed conservatively if it is small (up to $1 \mathrm{~cm}$ ) and shows no atypical clinical and dermoscopic features, can be followed-up every six months for the first 2 - 3 years and then just once a year. Large $(>1 \mathrm{~cm})$, ulcerated, rapidly changing, or atypical
Spitz nevus of childhood must be excised. Surgical excision is also recommended when Spitz nevus appear in the adulthood.

The limited numerousness of sampling is balanced by the long length of the follow-up, hardly performable on a larger sampling due to a low compliance especially in the late phases of the evolution of the lesion when the Spitz nevus, in its involutional phase, is not perceived by the patient as a potentially dangerous lesion to be inspected again.

\section{CONCLUSIONS}

Spitz nevus is a clinical entity with clinical ambiguity that makes problematic the diagnosis and the management of the patient.

On the basis of our observation the complete regression of the blue-white structures and the presence of multiphocal central hypopigmented areas might reflect a further evolutionary phase of pigmented Spitz nevus of childhood.

We support the hypothesis, yet described by other authors [6-8], that the globular and the starburst pattern and the homogeneous pigmentation might represent the natural evolution of the Spitz nevus in childhood rather than the expression of different clinicopathologic entities.

There are two extremes of management: Spitz nevus can be always periodically controlled, or should be always excised [9].

Based on this consideration, Spitz nevus appearing up to the age of 12 years can be easily diagnosed and managed conservatively if it is relatively small and shows no atypical clinical and dermoscopic features. Under these circumstances, a follow-up can be scheduled with control every 3 - 6 months [6]. In the absence of dramatic changes in colour, shape or size, such a follow-up protocol can be held until the appearance of a homogeneous pattern; since then, a 1-year follow-up can be employed [6].

The long term follow-up of Spitz nevus is recommended to avoid surgical excision of these lesions in childhood.

\section{REFERENCES}

[1] Pizzichetta, M.A., Argenziano, G., Grandi, G., et al. (2002) Morphologic changes of a pigmented Spitz nevus assessed by dermoscopy. Journal of the American Academy of Dermatology, 47, 137-139. doi: $10.1067 / \mathrm{mjd} .2002 .122193$

[2] Argenziano, G., Scalvenzi, M., Staibano, S., et al. (1999) Dermatoscopic pitfalls in differentiating pigmented Spitz naevi from cutaneous melanomas. British Journal of Dermatology, 141, 788-793. 
doi:10.1046/j.1365-2133.1999.03150.x

[3] Ferrara, G., Argenziano, G., Soyer, H.P., et al. (2005) The spectrum of Spitz nevi: A clinicopathologic study of 83 cases. Archives of Dermatology, 141, 1381-1387. doi:10.1001/archderm.141.11.1381

[4] Gill, M., Cohen, J., Renwick, N., et al. (2004) Genetic similarities between Spitz nevus and Spitzoid melanoma in children. Cancer, 101, 2636-2640. doi:10.1002/cncr.20680

[5] Yan, A.C. and Smolinski, K.N. (2005) Melanocytic nevi: Challenging clinical situations in pediatric dermatology. Advances in Dermatology, 21, 65-80. doi:10.1016/i.yadr.2005.05.001

[6] Brunetti, B., Nino, M., Sammarco, E., et al. (2005) Spitz naevus: A proposal for management. Journal of the European Academy of Dermatology and Venereology, 19, 391-393. doi:10.1111/j.1468-3083.2004.01137.x

[7] Argenziano, G., Zalaudek, I., Ferrara, G., et al. (2007) Involution: The natural evolution of pigmented Spitz and Reed nevi? Archives of Dermatology, 143, 549-551.

[8] Nino, M., Brunetti, B., Delfino, S., et al. (2009) Spitz nevus: Follow-up study of 8 cases of childhood starburst type and proposal for management. Dermatology, 218, 48-51.

[9] Piccolo, D., Ferrari, A. and Peris, K. (2003) Sequential dermoscopic evolution of pigmented Spitz nevus in childhood. Journal of the American Academy of Dermatology, 49, 556-558. 